\title{
Clinical Utility of Polygenic Risk Score in Sleep Disorder
}

\author{
Young-Min Park \\ Department of Psychiatry, Ilsan Paik Hospital, Inje University College of Medicine, Goyang, Korea
}

\begin{abstract}
Recently, polygenic risk scores (PRS) using genome-wide association study (GWAS) data have attracted much attention. The present article aimed to review the literature on the relationship between sleep or sleep disorder and PRS. PRS for restless legs syndrome was a very useful marker for genetic risk and clinical characteristics. In addition, highest snoring-PRS decile had approximately twice the odds of reporting recent snoring and choking during sleep. Another study revealed that PRS for schizophrenia was associated with higher fast spindle amplitude, density, and intensity. In case of depression, REM sleep fragmentation was also associated with PRS for depressive symptoms in adolescents. Regarding bipolar disorder, the relationship between insomnia-PRS and bipolar II disorder and sleep-duration-PRS and bipolar I disorder have also been reported. Insomnia-PRS also predicted an earlier age of first cannabis use and increased the number of symptoms of cannabis use disorder. Given the small effect size in the SNP-based association, PRS is a very useful marker for clinical settings, as well as for diagnosis and treatment. In the future, PRS will be a useful tool for personalized medicine.

Key Words: Polygenic risk score; Sleep disorder; Sleep trait

Received: June 12, 2021 Revised: June 17, 2021 Accepted: June 17, 2021

Corresponding author: Young-Min Park, MD, PhD, Department of Psychiatry, Ilsan Paik Hospital, Inje University College of Medicine, 170 Juhwa-ro, Ilsanseo-gu, Goyang 10380, Korea.

Tel: 82-31-910-7260, Fax: 82-31-910-7268, E-mail: medipark@hanmail.net

(a) This is an Open Access article distributed under the terms of the Creative Commons Attribution Non-Commercial License (https://creativecommons.org/licenses/bync/4.0) which permits unrestricted non-commercial use, distribution, and reproduction in any medium, provided the original work is properly cited.
\end{abstract}

\section{INTRODUCTION}

Recently, polygenic risk scores (PRS) using genome-wide association study (GWAS) data have attracted much attention. PRS are produced using the odds ratios (ORs; patients vs. normal controls) from an existing GWAS as a discovery sample and applied to a GWAS of an independent target sample [1].

Several studies using PRS have been conducted in patients with schizophrenia and mood disorders. One study examined the use of PRS for schizophrenia (SPR-PRS) to predict treatment response to lithium in patients with bipolar disorder [2]. The results found that patients with higher SPR-PRS responded less to lithium. In another study, SPR-PRS and PRS for bipolar disorder (BD-PRS) were calculated for a normal population with high creativity [3]. The higher the creativity, the higher the SPR and BD-PRS relative to a normal population with lower creativity. Recently, PRS has been in the spotlight for personalized medicine. For example, some investigators described the clinical utility of SPR-PRS, BD-PRS, and PRS for major depressive disorder (MDD-PRS) in a depressed patient. If the patient had high SPR- and BD-PRS, and low MDD-
PRS, atypical antipsychotics can be selected as a first-line treatment. PRS have also been used in sleep research [1]. However, the research is not well known compared to schizophrenia and mood disorders.

The present article aimed to review the literature on the relationship between sleep or sleep disorder and PRS.

\section{RESTLESS LEGS SYNDROME AND POLYGENIC RISK SCORES}

Recently, one restless legs syndrome (RLS) study using PRS was published. PRS was calculated using the GWAS meta-analysis for RLS patients who participated in the UK Biobank [4]. The p-value threshold was set at 0.01 because it had the largest variance. The number of variants included in the RLS-PRS was 600,420 . Unlike GWAS results, PRS based on GWAS has greater clinical utility. Although GWAS only provides statistically different variant information between patients and the normal population, PRS allows us to identify the clinical characteristics of each individual or group. In the study, the higher the PRS in the target sam- 
ple, the lower the educational attention, and the lower the cognitive function [4]. PRS also showed positive correlations between neuroticism and all body fat, as well as percentage fat in the trunk, legs, and arms, and waist-to-hip ratio [4]. These results are consistent with a study that reported an association between high RLSPRS and an unhealthy lifestyle [5]. The results from this study were also consistent with an epidemiological study that identified specific characteristics associated with patients with RLS including normal weight, high activity, non-smoking status, and low alcohol intake [6].

When calculating PRS, one can analyze a large number of variants using the p-value threshold of the GWAS data. However, only a small number of target variants can be selected. For example, in one study, PRSs based on 20 association variants in the GWAS data were calculated, and according to these PRSs, the target sample was divided into several groups [7]. The OR for RLS risk of individuals in the highest risk group (PRS 99.5\% quantile) was 17.6 [7]. When the $25 \%$ quantile and the $75 \%$ quantile groups were compared, the OR for RLS risk was 59. As demonstrated, RLS-PRS is a very useful marker for genetic risk and clinical characteristics.

\section{OBSTRUCTIVE SLEEP APNEA AND POLYGENIC RISK SCORES}

A recent study investigated PRS using GWAS data from the UK Biobank to predict recent snoring and obstructive sleep apnea (OSA) in an Australian sample [8]. Snoring PRS was significantly associated with recent snoring. Participants in the highest snoring-PRS decile had approximately twice the odds of reporting recent snoring and choking or struggling to breathe during sleep compared with those in the lowest decile. These findings suggest that PRS is a very useful marker for predicting OSA.

\section{SLEEP TRAIT AND POLYGENIC RISK SCORES}

In a study using UK Biobank data, PRS based on 78 SNPs related to long sleep duration were calculated [9]. Each participant's PRS was produced by summing the OR of risk alleles at each genetic variant which were weighted by the respective allelic effect sizes on longer sleep duration. Phenotypic variance in sleep duration showed a $1.4 \%$ variance. After adjusting for age, sex, and principal components, PRS was associated with obesity, congestive hypertension, heart failure, insomnia, and RLS. The PRS based on a focused set of variants with self-reported sleep duration in the Biobank confirmed the clinical utility of this score as a biological marker to predict sleep duration.

In another study, SPR-PRS was associated with higher fast spindle amplitude, density, and intensity [10]. PRS was calculated using ORs and p-values from data of the Psychiatric Genomics Consortium (PGC) GWAS dataset, which included 38,131 schizophrenia cases and 114,674 controls. A positive association between the genetic variants of schizophrenia and sleep spindle activity among healthy adolescents supported the hypothesis that sleep spindles and schizophrenia share similar genetic pathways. These findings were not consistent with the traditional hypothesis that patients with schizophrenia are associated with lower sleep spindle activity than normal controls $[11,12]$. This study indicated that altered sleep spindle activity may serve as a biological marker of schizophrenia and raised a new assumption that contradicted existing research evidence.

Some investigators have revealed that REM sleep fragmentation is associated with PRS for depressive symptoms in adolescents [13]. PRS was calculated using data from the Cohorts for Heart and Aging Research in Genomic Epidemiology (CHARGE) Consortium GWAS for three dimensions of depressive symptoms [14]. The dimensions were three sub-items from the Center for Epidemiologic Studies Depression Scale (CES-D): negative emotion, lack of positive emotion, and somatic complaints. The p-value threshold was set at 0.01 . The number of SNPs included in the PRS was 5,563, 5,575, 5,823, and 5,587 variants for positive affect, negative affect, and somatic and total scores, respectively. Depressive symptoms and PRS for somatic complaints were independently associated with more fragmented REM sleep. These findings suggested that REM fragmentation may be a distinct mechanism that lowers the quality of sleep and was associated with PRS for depressive symptoms.

The relationship between insomnia-PRS and bipolar II disorder (BIID) and sleep-duration-PRS and bipolar I disorder (BID) have also been reported. PRS was calculated using data on insomnia, sleep duration, daytime sleepiness from GWAS data in the UK Biobank [15]. Insomnia-PRS was associated with an increased relative risk of BIID compared to normal controls and BID. Sleepduration PRS was also associated with an increased relative risk of BID compared with BIID. Daytime-sleepiness-PRS was associated with both BID and BIID. In contrast, the relative risk between BD subtypes for daytime-sleepiness-PRS did not differ significantly, unlike insomnia-PRS and sleep-duration PRS. In addition, "morningness"-PRS was associated with a reduced relative risk of BID and BIID compared with normal controls. This study suggests that the genetic liability of sleep traits of BID and BIID, as well as that of $\mathrm{BD}$ and normal controls differ from each other.

One study examined the predictive response of PRS to therapeutic sleep deprivation (SD) [16]. PRS was produced using GWAS data from the PGC. The PRS was significantly higher in non-responders to SD than in normal controls and higher PRS in nonresponders than in responders to $\mathrm{SD}$, although the differences were not statistically significant. These findings suggest that by applying the therapeutic SD based on genetic liability, such as PRS, a better understanding of the mechanisms of depression may be gained.

\section{SLEEP DISORDER, COMORBIDITY, AND POLYGENIC RISK SCORES}

Some investigators have revealed the relationship between PRS 
based on sleep-related GWAS and cannabis use in a target sample [17]. Insomnia-PRS predicted an earlier age of first cannabis use and increased the number of symptoms of cannabis use disorder. However, the short sleep duration and "eveningness" chronotype PRS did not significantly predict any of the cannabis variables in the target sample.

Another PRS study has suggested that narcolepsy is genetically associated with attention-deficit/hyperactivity disorder (ADHD) [18]. The discovery sample for narcolepsy consisted of 1,562 normal controls and 409 patients with narcolepsy. The narcolepsy-PRS was significantly associated with hyperactivity, inattention, and total scores on the attention deficit hyperactivity disorder rating scale. However, essential hypersomnia-PRS was not significantly associated with either domain of ADHD traits. The results indicated that there may be a shared genetic background in ADHD and narcolepsy. These findings suggest a shared genetic background in ADHD and narcolepsy.

\section{CONCLUSIONS}

Given the small effect size in the SNP-based association, PRS is a very useful marker for clinical settings, as well as for diagnosis and treatment. In the future, PRS will be a useful tool for personalized medicine.

\section{Acknowledgments}

This study was supported by a grant from the National Research Foundation of Korea (NRF), funded by the Ministry of Science and ICT (2020R1F1A1073188).

\section{Conflicts of Interest}

The author has no potential conflicts of interest to disclose.

\section{ORCID iD}

Young-Min Park

https://orcid.org/0000-0002-4993-1426

\section{REFERENCES}

1. Ikeda M, Saito T, Kanazawa T, Iwata N. Polygenic risk score as clinical utility in psychiatry: a clinical viewpoint. J Hum Genet 2021;66:53-60.

2. International Consortium on Lithium Genetics (ConLi+Gen). Association of polygenic score for schizophrenia and HLA antigen and inflammation genes with response to lithium in bipolar affective disorder: a genome-wide association study. JAMA Psychiatry 2018;75:65-74.

3. Li H, Zhang C, Cai X, Wang L, Luo F, Ma Y, et al. Genome-wide association study of creativity reveals genetic overlap with psychiatric disorders, risk tolerance, and risky behaviors. Schizophr Bull 2020;46:1317-1326.

4. Didriksen M, Nawaz MS, Dowsett J, Bell S, Erikstrup C, Pedersen OB, et al. Large genome-wide association study identifies three novel risk variants for restless legs syndrome. Commun Biol 2020;3:703.

5. Didriksen M, Rigas AS, Allen RP, Burchell BJ, Di Angelantonio E, Nielsen $\mathrm{MH}$, et al. Prevalence of restless legs syndrome and associated factors in an otherwise healthy population: results from the Danish Blood Donor Study. Sleep Med 2017;36:55-61.

6. Batool-Anwar S, Li Y, De Vito K, Malhotra A, Winkelman J, Gao X. Lifestyle factors and risk of restless legs syndrome: prospective cohort study. J Clin Sleep Med 2016;12:187-194.

7. Schormair B, Zhao C, Bell S, Tilch E, Salminen AV, Pütz B, et al. Identification of novel risk loci for restless legs syndrome in genome-wide association studies in individuals of European ancestry: a meta-analysis. Lancet Neurol 2017;16:898-907.

8. Campos AI, García-Marín LM, Byrne EM, Martin NG, Cuéllar-Partida G, Rentería ME. Insights into the aetiology of snoring from observational and genetic investigations in the UK Biobank. Nat Commun 2020;11:817.

9. Dashti HS, Redline S, Saxena R. Polygenic risk score identifies associations between sleep duration and diseases determined from an electronic medical record biobank. Sleep 2019;42:zsy247.

10. Merikanto I, Utge S, Lahti J, Kuula L, Makkonen T, Lahti-Pulkkinen M, et al. Genetic risk factors for schizophrenia associate with sleep spindle activity in healthy adolescents. J Sleep Res 2019;28:e12762.

11. Manoach DS, Demanuele C, Wamsley EJ, Vangel M, Montrose DM, Miewald J, et al. Sleep spindle deficits in antipsychotic-naïve early course schizophrenia and in non-psychotic first-degree relatives. Front Hum Neurosci 2014;8: 762.

12. Ferrarelli F, Peterson MJ, Sarasso S, Riedner BA, Murphy MJ, Benca RM, et al. Thalamic dysfunction in schizophrenia suggested by whole-night deficits in slow and fast spindles. Am J Psychiatry 2010;167:1339-1348.

13. Pesonen AK, Gradisar M, Kuula L, Short M, Merikanto I, Tark R, et al. REM sleep fragmentation associated with depressive symptoms and genetic risk for depression in a community-based sample of adolescents. J Affect Disord 2019;245:757-763.

14. Demirkan A, Lahti J, Direk N, Viktorin A, Lunetta KL, Terracciano A, et al. Somatic, positive and negative domains of the Center for Epidemiological Studies Depression (CES-D) scale: a meta-analysis of genome-wide association studies. Psychol Med 2016;46:1613-1623.

15. Lewis KJS, Richards A, Karlsson R, Leonenko G, Jones SE, Jones HJ, et al. Comparison of genetic liability for sleep traits among individuals with bipolar disorder I or II and control participants. JAMA Psychiatry 2020;77:303310.

16. Trautmann N, Foo JC, Frank J, Witt SH, Streit F, Treutlein J, et al. Response to therapeutic sleep deprivation: a naturalistic study of clinical and genetic factors and post-treatment depressive symptom trajectory. Neuropsychopharmacology 2018;43:2572-2577.

17. Winiger EA, Ellingson JM, Morrison CL, Corley RP, Pasman JA, Wall TL, et al. Sleep deficits and cannabis use behaviors: an analysis of shared genetics using linkage disequilibrium score regression and polygenic risk prediction. Sleep 2021;44:zsaa188.

18. Takahashi N, Nishimura T, Harada T, Okumura A, Choi D, Iwabuchi T, et al. Polygenic risk score analysis revealed shared genetic background in attention deficit hyperactivity disorder and narcolepsy. Transl Psychiatry 2020; 10:284. 